\title{
LETTER
}

\section{Noise levels produced by high-frequency oscillation}

\author{
Matt PG Morgan* and Nicki Haskins
}

High-frequency oscillation is now an accepted treatment for patients with severe respiratory failure. Although the technology has advanced in recent times, the noise levels produced by such devices remain high. Exposure to excessive noise in intensive care may cause hearing problems for both staff and patients following long-term exposure.

We investigated the noise level in decibels produced by a SensorMedics 3100B ventilator (SensorMedics, Bilthoven, the Netherlands). The maximum total noise level recorded was 85 decibels, with an average of 75 decibels over a 12 -hour period. These daily noise levels exceed the minimum required for action to be taken by employers according to regulations operating within the United Kingdom.

We suggest three strategies for dealing with this issue that aim to reduce potential harm to both patients and staff. First, reduce the noise produced by oscillators. This should be done in conjunction with equipment manufacturers. Second, reduce the potential for damage to patient's hearing by the provision of ear protection and minimise ototoxic drug use whilst oscillation is employed. Although this may lead to difficulties in patient communication, in reality those receiving high-frequency oscillation are normally deeply sedated and paralysed. Finally, reduce the risks to staff working with these patient groups. The provision of staff ear protection would be problematic, so other strategies should be used. These could include mandatory training, limiting time spent nursing oscillated patients using rotational staffing policies, and excluding those with pre-existing hearing problems.

\section{Competing interests}

The authors declare that they have no competing interests.

Published: 28 April 2010

doi:10.1186/cc8958

Cite this article as: Morgan MPG, Haskins N: Noise levels produced by highfrequency oscillation. Critical Care 2010, 14:415.
*Correspondence: mattmorgan@me.com

Department of Critical Care, University Hospital of Wales, Heath Park, Cardiff, CF14 4XW UK 